\title{
Supernovae and Positron Annihilation Radiation
}

\author{
P.A. Milne ${ }^{a}$ J.D. Kurfess, R.L. Kinzer ${ }^{b}$ M.D. Leising ${ }^{c}$ \\ ${ }^{a}$ NRC/NRL Resident Research Associate, Naval Research Lab, Code 7650, \\ Washington DC 20375 \\ ${ }^{\mathrm{b}}$ Naval Research Lab, Code 7650, Washington DC 20375 \\ ${ }^{\mathrm{c}}$ Clemson University, Clemson, SC 29631
}

\begin{abstract}
Radioactive nuclei, especially those created in SN explosion, have long been suggested to be important contributors of galactic positrons. In this paper we describe the findings of three independent OSSE/SMM/TGRS studies of positron annihilation radiation, demonstrating that the three studies are largely in agreement as to the distribution of galactic annihilation radiation. We then assess the predicted yields and distributions of SN-synthesized radionuclei, determining that they are marginally compatible with the findings of the annihilation radiation studies.
\end{abstract}

Key words: Supernovae, ISM

\section{OSSE/SMM/TGRS Observations}

Nine years of observations made with the Oriented Scintillation Spectrometer Experiment (OSSE) on-board NASA's COMPTON observatory (19912000)[1], eight years of observations made with the Gamma-Ray Spectrometer on-board the Solar Maximum Mission (SMM) (1980-1989)[2], and two years of observations made with the Transient Gamma-Ray Spectrometer (TGRS) on-board the WIND mission (1995-1997) [3] have been utilized to study the galactic distribution of positron annihilation radiation. The OSSE instrument featured a $3.8^{\circ} \times 11.4^{\circ} \mathrm{FWHM} \mathrm{FoV}$, a $\sim 3 \times 10^{-5}$ photons $\mathrm{cm}^{-2} \mathrm{~s}^{-1}$ line sensitivity $\left(10^{6} \mathrm{~s}\right.$ on-source time), and a $45 \mathrm{keV}$ energy resolution at $511 \mathrm{keV}$. These detector attributes have permitted the first detailed studies of the distribution of annihilation radiation in the inner radian of the Galaxy. The annihilation of positrons with electrons gives rise to two spectral features, a line emission at $511 \mathrm{keV}$ and a positronium continuum emission (which increases in intensity with energy roughly as a power law up to $511 \mathrm{keV}$ and falls abruptly to zero above $511 \mathrm{keV}$ )[4]. The TGRS instrument, which featured a germanium detector with excellent energy resolution, has demonstrated that the integrated flux from the inner radian is best described as a narrow $511 \mathrm{keV}$

Preprint submitted to Elsevier Preprint 24 October 2018 
line $(\mathrm{FWHM} \leq 1.8 \mathrm{keV})$ and a positronium continuum to $511 \mathrm{keV}$ line ratio of $\sim 3.6$ (which corresponds to a positronium fraction of $\mathrm{f}_{P s}=0.94$ ) [3].

Purcell et al. (1997) [5] reported results from combined OSSE/SMM/TGRS studies of the $511 \mathrm{keV}$ line component of annihilation radiation. They found the $511 \mathrm{keV}$ emission to be comprised of three components; 1) an intense bulge emission, 2) a fainter disk emission, and 3) an enhancement of emission at positive latitudes (hereafter called a PLE). The PLE was also reported by Cheng et al. (1997) [6], and has been interpreted to be an "annihilation fountain" by Dermer \& Skibo[7]. Purcell et al. (1997) characterized the emission via mapping, employing the SVD matrix inversion algorithm, and via model fitting, testing the combination of a spheroidal Gaussian bulge, a disk that is flat in longitude to $\pm 40^{\circ}$ and Gaussian in latitude $\left(\mathrm{FWHM}=9^{\circ}\right)$, and a spheroidal PLE. The two characterizations differ in the thickness of the Gaussian disk (SVD being narrower) and the extension of the PLE. The enhancement of the PLE varied from $1.5 \times 10^{-4}$ phot $\mathrm{cm}^{-2} \mathrm{~s}^{-1}$ for the SVD map to $9 \times 10^{-4}$ phot $\mathrm{cm}^{-2} \mathrm{~s}^{-1}$ for the 2D Gauss. PLE $\left(\mathrm{FWHM}=16.4^{\circ}\right)$. The positron annihilation rates suggested by these two approaches are $(4.2 \pm 0.5) \times 10^{43} \mathrm{e}^{+} \mathrm{s}^{-1}$ (SVD) and $(3.3 \pm 0.50) \times 10^{43} \mathrm{e}^{+} \mathrm{s}^{-1}$ (2D Gauss.), with B/D ratios of 0.5 and 0.3 .

Parallel studies were performed by Kinzer et al. (2001). Those studies investigated line and continuum positron annihilation radiation from the galactic plane, finding that the two emissions are similarly distributed within the statistical precision of the data. Kinzer et al. (2001) suggest two spatial distributions to explain the OSSE/SMM observations. The first model is comprised of a 2D Gaussian bulge $\left(4.9^{\circ} \pm 0.7^{\circ}(b) \times 6.3^{\circ} \pm 1.5^{\circ}(l)\right)$, and two disks; a 2D Gaussian disk $\left(12^{\circ}(b) \times 35^{\circ} \pm 10^{\circ}(l)\right)$ and a widely-distributed CO disk (also with $12^{\circ}$ latitudinal thickness). The second model is comprised of a slightly ellipsoidal bulge which follows the " $\mathrm{R}^{1 / 4}$ " distribution function and an exponential disk (refer to Kinzer et al. (2001) for details of these functions). The positron annihilation rates suggested by these models are $3.9 \pm 0.4 \times 10^{43} \mathrm{e}^{+} \mathrm{s}^{-1}$ and $3.1 \pm 0.4$ $\mathrm{x} 10^{43} \mathrm{e}^{+} \mathrm{s}^{-1}$ respectively, with $\mathrm{B} / \mathrm{D}$ ratios of 0.7 and 3 .

We report here updates from our continuing analysis which extends the study of Purcell et al. (1997) (see also Milne et al. $(1998,1999)[9,13])$. The primary differences between current studies and Purcell et al. (1997) are; 1) the inclusion of more observations, both archival and data collected after Purcell et al. (1997), and 2) reporting maps of the positronium continuum emission in addition to the $511 \mathrm{keV}$ line. To extract the positronium continuum component from the underlying galactic continuum emission, we widened the spectral modeling to include thermal bremsstrahlung and exponentially-truncated power-law models. We also removed high-energy diffuse continuum emission following a prescription from Kinzer et al. (1999), distributing the emission spectrally according to a power-law $(\alpha=-1.65)$ and spatially according to a $90^{\circ} \times 5^{\circ} 2 \mathrm{D}$ Gaussian[11]. 


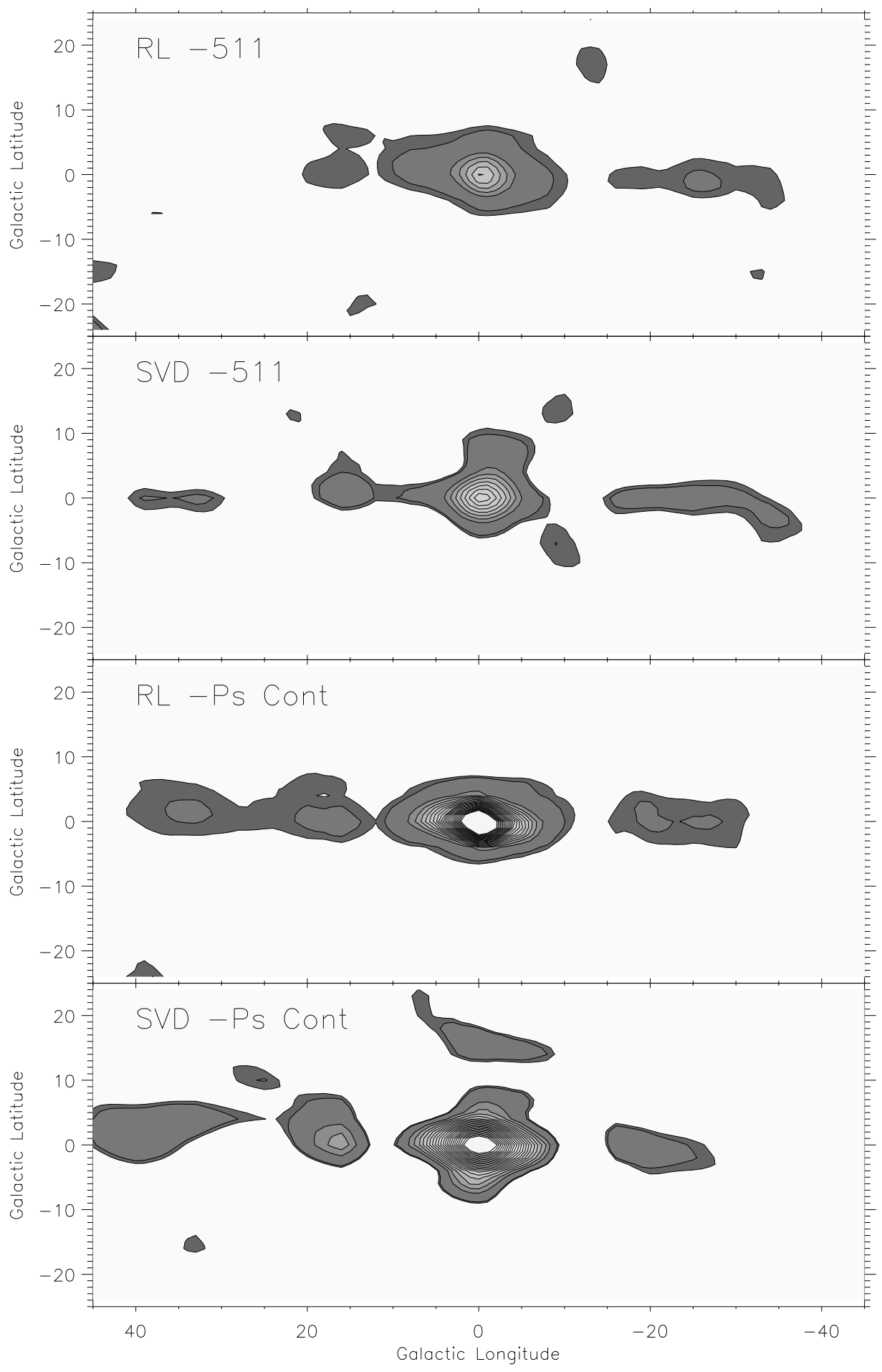

Fig. 1. Four characterizations of positron annihilation radiation from mapping algorithms. The upper two panels are Richardson-Lucy and SVD maps of $511 \mathrm{keV}$ line emission. The lower two panels are Richardson-Lucy and SVD maps of positronium continuum emission. The SVD mapping algorithm was used in Purcell et al. (1997). 
Maps made from mapping algorithms applied to the resulting data-set are shown in Figure 1. The top two panels show Richardson-Lucy and SVD maps of $511 \mathrm{keV}$ line emission, the lower two panels show Richardson-Lucy and SVD maps of positronium continuum emission. Although not identical, the four maps are similar in appearance, with all exhibiting an intense bulge emission and a fainter planar emission. 1 Pairings of bulge and disk components suggest the same families of solutions for both the line and positronium continuum emissions. Both suggest that the bulge-to-disk ratio can vary from $0.2-3.3$ depending upon whether the bulge component features a halo (which leads to a large B/D). All of these comparisons support the Kinzer et al. (2001) suggestion that the two annihilation components are similarly distributed. Two bulge-disk combinations that span the range of favored solutions are shown in Figure 2. The upper panel shows a "bulge-dominated" solution, where a halo bulge plus a thin disk combine to annihilate $4.1 \times 10^{43} \mathrm{e}^{+} \mathrm{s}^{-1}$ with a $\mathrm{B} / \mathrm{D}=3.3$. The lower panel shows a "disk-dominated" solution, where a 2D Gaussian bulge (without a halo) plus a thick disk combine to annihilate $4.1 \times 10^{43} \mathrm{e}^{+} \mathrm{s}^{-1}(\mathrm{~B} / \mathrm{D}=0.2)$.

\section{Supernovae and Galactic Positrons}

The $\mathrm{B} / \mathrm{D}$ ratios favored by the current work bracket those found by Purcell et al. (1997) and Kinzer et al. (2001), allowing a range of $0.2 \leq \mathrm{B} / \mathrm{D} \leq$ 3.3. The positron annihilation rate is suggested to lie within the range of (3.1 -4.2) x $10^{43} \mathrm{e}^{+} \mathrm{s}^{-1}$. To compare suggested SN positron production rates with observations, a number of ad-hoc assumptions must be made. First, we assume that positrons do not travel large distances from their site of origin, so that source B/D ratios are assumed to equal observed annihilation radiation $\mathrm{B} / \mathrm{D}$ ratios. Second, we assume that there is no leakage of positrons from the Galaxy nor enrichment of positrons from extra-galactic sources. Third, we assume that the number of galactic positrons is not changing, that there is a steady-state population of positrons. These three assumptions allow us to compare annihilation radiation fluxes with positron production rates. Fourth, we assume that the SNe in the Galaxy scale with B-band luminosity the same way as SNe in other late spiral galaxies. This permits the use of extra-galactic $\mathrm{SN}$ rates as an indicator of the recent SN activity in the Galaxy.

SNe Ia produce positrons primarily from the decay of ${ }^{56} \mathrm{Ni} \rightarrow{ }^{56} \mathrm{Co} \rightarrow{ }^{56} \mathrm{Fe}$, although the decay of ${ }^{44} \mathrm{Ti} \rightarrow{ }^{44} \mathrm{Sc} \rightarrow{ }^{44} \mathrm{Ca}$ also contributes positrons. Chan \& Lingenfelter (1993) simulated positron transport through SN Ia models and suggested that $\sim 5 \%$ of ${ }^{56} \mathrm{Co}$-decay positrons escape the ejecta for favorable magnetic field scenarios. Milne et al. (1999,2001a) followed up that study by comparing SN Ia light curves with simulations. That study found that the

$\overline{1}$ We refer the reader to Milne et al. 2001 for a discussion of current investigations of the PLE. 

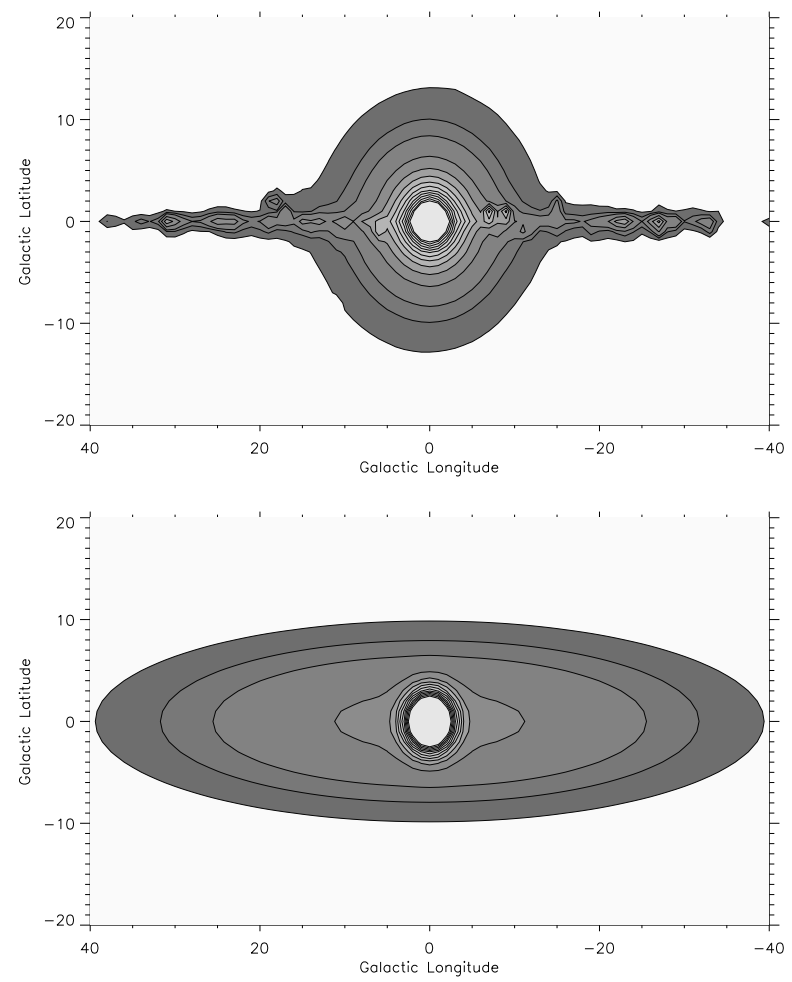

Fig. 2. Two characterizations of positron annihilation radiation from model-fitting. The upper panel shows a "bulge-dominated" solution. The lower panel shows a "disk-dominated" solution. The "bulge-dominated" solution is similar to the nova-spheriod model of Kinzer et al. (2001), the "disk-dominated" solution is similar to the three 2D Gaussian model of Purcell et al. (1997) and to the two 2D Gaussian + CO model of Kinzer et al. (2001).

light curves suggest that $\sim 8 \times 10^{52}$ positrons escape the ejecta from a normal type Ia supernova. Assuming the typical ${ }^{44} \mathrm{Ti}$ yield in a SN Ia to be $2 \times 10^{-5}$ $\mathrm{M}_{\odot}$, and $100 \%$ escape of those positrons, only $5 \times 10^{50}{ }^{44} \mathrm{Ti}$ positrons would be produced in a single SN Ia. Assuming the SN Ia rate in the Galaxy to be 0.4 SN Ia per century, this translates to $1.0 \times 10^{43}$ and $6 \times 10^{40}$ positrons s $^{-1}$ from ${ }^{56} \mathrm{Co}$ and ${ }^{44} \mathrm{Ti}$ decays. The SN Ia contribution to galactic positrons principally depends upon whether positrons escape in quantity from the SN ejecta.

Estimating the massive star (SNII/Ib and WR star) contribution to galactic positrons is more complicated. They produce positrons through the decays of ${ }^{44} \mathrm{Ti} \rightarrow{ }^{44} \mathrm{Sc} \rightarrow{ }^{44} \mathrm{Ca}$, and ${ }^{26} \mathrm{Al} \rightarrow{ }^{26} \mathrm{Mg}$, the latter from both hydrostatic and explosive phases. The predicted yields of these isotopes vary dramatically in individual events. Woosley \& Weaver (1995) suggest that they range from $\leq 10^{-6}$ up to $2.3 \times 10^{-4} \mathrm{M}_{\odot}$ for ${ }^{44} \mathrm{Ti}$, and $1.7 \times 10^{-5}-3.6 \times 10^{-4} \mathrm{M}_{\odot}$ for ${ }^{26} \mathrm{Al}[16]$. SN II/Ib rates have been reported to range between $1.5-3.3 \mathrm{SNe} \mathrm{II} / \mathrm{Ib}$ per century[15,21]. To help to constrain our estimates, we use observations of 1.809 MeV line emission, which is produced in $100 \%$ of ${ }^{26} \mathrm{Al}$ decays. These suggest about $3 \times 10^{42}{ }^{26} \mathrm{Al}$ decays $\mathrm{s}^{-1}$ occur entirely in the galactic disk (see 
Pluschke in these proceedings) supplying $2.4 \times 10^{42}$ positrons $\mathrm{s}^{-1}$.

The positron contribution of ${ }^{44} \mathrm{Ti}$ is best constrained by galactic chemical evolution and the solar abundance of the daughter ${ }^{44} \mathrm{Ca}$. This requires that $2-4 \times 10^{-6} \mathrm{M}_{\odot}$ of ${ }^{44} \mathrm{Ti}$ are now ejected into the galactic disk [17]. We note, however, that the production obtained by multiplying the above rates and yields falls short of this. The implied positron production rate is $1.6-3.2 \mathrm{x}$ $10^{42} \mathrm{~s}^{-1}$, and we estimate the total massive star contribution to the disk to be $4-6 \times 10^{42}$ positrons $\mathrm{s}^{-1}$.

Combining these estimates, $30 \%-50 \%$ of galactic positrons may be explained by thermonuclear SNe and massive stars. This appears promising, but this model must also agree with the observed $\mathrm{B} / \mathrm{D}$ ratio of annihilation radiation. SNe Ia occur in both bulges and disks of spiral galaxies, with suggested B/D ratios ranging from $1 / 2$ to $1 / 7[20,21]$. Our estimates suggest that the $\mathrm{B} / \mathrm{D}$ ratio ranges from 0.1 to 0.6 . These values are at the low extreme of the fitted $\mathrm{B} / \mathrm{D}$ ratios from the annihilation data (which are realized when halo-less bulges are combined with thick disks). If ${ }^{56} \mathrm{Co}$ positrons do not escape from the ejecta of SNe Ia, in contrast to the studies of Milne et al. $(1999,2001)$, then the massive star contribution is at most $20 \%$ of galactic positrons, and the bulge component is entirely due to a different type of source.

\section{Discussion}

After accepting a couple of $a d-h o c$ assumptions, we have shown that SNe are likely to be a dominant contributor to galactic positrons. We find that the maximum $\mathrm{SN}+{ }^{26} \mathrm{Al}$ contribution occurs with low $\mathrm{B} / \mathrm{D}$ ratios, requiring that the remaining $45 \%$ of positrons be produced by a wholly-bulge source type. Lowering the SN II/Ib contribution somewhat relaxes this requirement, but the remaining sources must collectively have a large B/D ratio. Potential sources include; 1) a recent galactic center starburst that produced a population of bulge positrons through massive star nucleosynthesis and SNe II, 2) positrons produced by a galactic center compact source, 3) nucleosynthesis in novae. All three of these sources are contrained by theory or observations. The starburst is constrained by the absence of a bulge component in the 1.809 $\mathrm{MeV}$ maps and the failure to detect $1.173 \mathrm{MeV}$ emission from the decay of SN-produced ${ }^{60} \mathrm{Fe}$. OSSE observations of $511 \mathrm{keV}$ line emission from the galactic center limit the contribution of a compact source to less than $1.5 \times 10^{-4}$ photons $\mathrm{cm}^{-2} \mathrm{~s}^{-1}[10]$. This is less than $6 \%$ of the total annihilation radiation. However, if the positrons diffuse throughout the bulge, a GC compact source could contribute at a higher level. Novae production of positrons from radionuclei (other than ${ }^{26} \mathrm{Al}$ ) are constrained by simulations that suggest that few positrons produced in $\mathrm{CO}$ novae would escape [22], and low ${ }^{22} \mathrm{Na}$ yields in ONe novae[23]. 
There are too many uncertainties involved in these comparisons to determine the validity of any of the assumptions that were used. Advances in the studies of the gamma-ray and optical emission from SNe will eventually resolve these

questions. At present, we must be content with interpreting hints as to the SN contribution to positrons in the Galaxy.

\section{References}

[1] Johnson, W.N., et al. ApJS, 86, 693 (1993).

[2] Share, G.H., et al., ApJ 326, 717 (1988).

[3] Harris, M.J., et al., ApJ 501, L55 (1998).

[4] Ore, A. \& Powell, J.L., Phys Rev, 75, 11 (1949).

[5] Purcell, W.R., et al., ApJ 491, 725 (1997).

[6] Cheng, L.-X., et al., ApJ 481, L43 (1997).

[7] Dermer, C.D. \& Skibo, J.G., ApJ 487, L57 (1997).

[8] Kinzer, R.L., et al., ApJ, 559, 705 (2001).

[9] Milne, P.A. et al., Astro. Lett. \& Comm. 38, 441 (1998).

[10] Milne, P.A. et al., in Proceedings of the 6th Compton Symposium, in press (astro-ph 0106157) (2001a).

[11] Kinzer, R.L., Purcell W.R., \& Kurfess, J.D., ApJ 515, 215 (1999).

[12] Chan, K.-W. \& Lingenfelter, R., ApJ, 405, 614 (1993).

[13] Milne, P.A., The, L.S., Leising, M.D., ApJS, 124, 503 (1999).

[14] Milne, P.A., The, L.S., Leising, M.D., ApJ-accepted, (astro-ph 0104185) (2001b).

[15] Cappellaro, E., Turatto, M., astro-ph, 0012455 (2000).

[16] Woosley, S.E., Weaver, T.A., ApJS, 101, 181 (1995).

[17] Timmes, F. X., Woosley, S. E., Hartmann, D. H., \& Hoffman, R. D., ApJ, 464, 332 (1996).

[18] Vink, J., et al. astro-ph, 0107468 (2001).

[19] Mahoney, W., Ling, J.C., Jacobson, A., ApJ, 286, 578 (1984).

[20] Howell, A., Wang, L., Wheeler, J.C., ApJ, 530, 166 (2000).

[21] Hatano, K., Fisher, A., Branch, D., MNRAS, 290, 360 (1997).

[22] Leising, M.D., \& Clayton, D.D., ApJ, 323, 159 (1987).

[23] Jose, J., Coc, A., \& Hernanz, M., ApJ, 520, 347 (1999). 